\title{
Influência do preço da mão de obra na sustentabilidade das explorações vitícolas durienses: uma aplicação de modelos baseados em agentes
}

\author{
The influence of labour costs on the sustainability of douro wine farms: an \\ application of $A B M$
}

\author{
João Matias ${ }^{1}$, Adelaide Cerveira² (D), Cátia Santos ${ }^{3}$ (D), Ana Alexandra Marta-Costa ${ }^{4}$ (D) \\ ${ }^{1}$ Centro de Matemática (CMAT-UTAD), Universidade de Trás-os-Montes e Alto Douro (UTAD), Vila Real, Portugal. E-mail: \\ j_matias@utad.pt \\ ${ }^{2}$ Instituto de Engenharia de Sistemas e Computadores, Tecnologia e Ciência (INESC-TEC), Universidade de Trás-os-Montes e \\ Alto Douro (UTAD), Vila Real, Portugal. E-mail: cerveira@utad.pt \\ ${ }^{3}$ COlab Vines\&Wines, Associação para o Desenvolvimento da Viticultura Duriense (ADVID) e Centro de Estudos \\ Transdisciplinares para o Desenvolvimento (CETRAD), Vila Real, Portugal. E-mail: catia.santos@advid.pt \\ ${ }^{4}$ Centro de Estudos Transdisciplinares para o Desenvolvimento (CETRAD), Universidade de Trás-os-Montes e Alto Douro \\ (UTAD), Vila Real, Portugal. E-mail: amarta@utad.pt
}

\begin{abstract}
Como citar: Matias, J., Cerveira, A., Santos, C. \& Marta-Costa, A. A. (2021). Influência do preço da mão de obra na sustentabilidade das explorações vitícolas durienses: uma aplicação de modelos baseados em agentes. Revista de
\end{abstract} Economia e Sociologia Rural, 59(1), e238886. https://doi.org/10.1590/1806-9479.2021.238886

Resumo: Em Portugal, a disponibilidade de mão de obra tem-se revelado um fator-chave para a viticultura de montanha. Estatísticas mais recentes denotam valores preocupantes que poderão colocar em causa a produção de vinho de qualidade e o atraente conjunto de paisagens vitivinícolas consideradas um recurso potencial para o desenvolvimento do turismo. Por a região duriense ser uma das principais regiões vitivinícolas portuguesas, caracterizada por proeminente e acentuada viticultura de montanha, pretende-se, neste trabalho, simular o comportamento das suas explorações perante alterações do preço de mão de obra, recorrendo-se a modelos baseados em agentes (ABM). Foi ainda usado o software MATLAB para obter funções periódicas ajustadas aos dados caracterizadores das variáveis consideradas pertinentes, obtidas de inquéritos presenciais a 110 explorações e atendendo aos dados disponibilizados pela Rede de Informação de Contabilidades Agrícolas (RICA). Posteriormente, o software ABM (NETLOGO) foi selecionado para simular os próximos 100 anos, familiarizando a dinâmica real baseada nos dados anteriormente considerados. Dependendo do preço da mão de obra, no final do horizonte de simulação, com o preço da uva a 0,77€ / kg, das 300 explorações existentes inicialmente, sobrevivem entre 127 e 231 (42,3\% a 77\%). Num cenário mais otimista, com o preço da uva a 1,17 €/kg a taxa de sobrevivência oscila entre $72,1 \%$ e $93,2 \%$.

Palavras-chave: agent-based model, setor vitivinícola, desempenho, sustentabilidade, mão de obra.

\begin{abstract}
In Portugal, labour availability has been revealed as a key factor for the activity, particularly in mountain viticulture. The latest statistics present worrying values that could undermine the production of quality wine and the attractive set of wine landscapes considered as a potential resource for tourism development. The Douro Region is one of the main Portuguese wine regions, characterized by a prominent and accentuated mountain viticulture. This paper aims to simulate the behaviour of its farms about the changes in the price of labour, through Agent-Based Models (ABM). The MATLAB software was used to obtain periodic functions adjusted to the data that characterize the relevant variables, obtained from face-to-face surveys of 110 farms, and taking into account the data provided by PTFADN. Subsequently, the ABM software (NETLOGO) was selected to simulate the next 100 years, familiarizing the real dynamics based on the previously considered data. Depending on the price of labour at the end of the simulation horizon, with a grape price of $0,77 € / \mathrm{kg}$, from the 300 initially existing farms survive
\end{abstract}


between 127 and 231 (42,3 - 77\%). In a more optimistic scenario, with a grape price of 1,17 €/kg, the survival rate ranges between 72.1 and $93.2 \%$.

Keywords: agent-based model, wine sector, performance, sustainability, labour.

\section{INTRODUÇÃO}

A demografia e a dinâmica do mercado de trabalho são reconhecidas como fatoreschave para o desenvolvimento de uma região e das suas atividades econômicas, como é o caso da produção de uva para a produção de vinho (Rebelo, 2018; Santos et al., 2019).

Embora tenham havido muitas melhorias agrícolas nos últimos anos em razão da maciça mecanização e dos avanços tecnológicos, essa atividade ainda exige quantidade razoável de mão de obra. Esse requisito é mais pronunciado na viticultura íngreme nas montanhas, o que aumenta as exigências de mão de obra (Moreira \& Guedes de Pinho, 2011). Tal preocupação é muito proeminente na região demarcada do Douro (RDD), localizada no nordeste de Portugal, onde as vinhas em declives acentuados são dominantes, mas que reúnem condições naturais que propiciam produção de vinhos de qualidade e o famoso vinho do Porto, conhecidos internacionalmente. A RDD encerra $22,4 \%$ das áreas com vinha em Portugal, correspondentes a 42.556 ha (Instituto da Vinha e da Vinho, 2018).

O setor vitivinícola português representa um grande volume de negócios e valor agregado e, acima de tudo, um valor muito positivo na sua balança comercial, permitindo que o país ocupe o 110 lugar no ranking mundial e o 5o no grupo de produtores europeus. Em 2018, a produção atingiu cerca de 6 milhões de hectolitros, correspondendo a 2,2\% da produção mundial, apesar de se registar um decréscimo de 10\% relativamente a 2017 (International Organization of Vine \& Wine, 2019). Porém, se ao nível econômico a atividade apresenta uma importância direta inegável, as vinhas do país desenham um atraente conjunto de paisagens vitivinícolas consideradas um recurso que muito potencializa o desenvolvimento do turismo (Rachão e Joukes, 2017). Essa relação positiva entre o setor vitivinícola e a competitividade regional e os seus efeitos no ambiente passa, ainda, por seu contributo no desenvolvimento de infraestruturas, na melhoria do bem-estar social geral e na oferta de empregos (Hall \& Sharples, 2008; Viassone et al., 2016). Em Portugal, o negócio vitivinícola é responsável por $8 \%$ do emprego à população (Instituto Nacional de Estatística, 2017b).

Nesse contexto, há grande preocupação com o crescimento sustentável do setor, assente na abordagem triple bottom line, que interliga o desempenho econômico e as dimensões ambiental e social (Marta-Costa et al., 2012; Mencarelli \& De Propris, 2014).

Flint et al. (2011) definem a sustentabilidade como a capacidade de um negócio ser bemsucedido a longo prazo e indicam, especificamente, a resiliência desse negócio às forças competitivas do mercado.

Um dos fatores na RDD que muito poderá afetar a sustentabilidade da sua viticultura é a necessidade e a disponibilidade de mão de obra. O mercado de trabalho está intimamente relacionado com a dinâmica demográfica e, nessa região, houve redução substancial da força de trabalho disponível acima de 5\%, entre 2011 e 2016 (Instituto Nacional de Estatística, 2017b). Tal fato é particularmente explicado com o êxodo dos jovens para as áreas urbanas (Van Der Laan, 2016) e os baixos salários agrícolas, levando os trabalhadores disponíveis a procurar atividades mais bem remuneradas que aumentam simultaneamente seu prestígio social (Marta-Costa, 2010; Santos et al., 2019). Rebelo (2018) considera essa tendência uma das principais ameaças e desafios que a RDD enfrentará no futuro próximo. Espera-se que esse problema e tendência se intensifiquem nos próximos anos, com a expectativa de uma população ativa decadente de 2,8\% para 2021 (Santos et al., 2019).

Neste sentido, torna-se imperativo simular tendências no comportamento do setor vitivinícola a longo prazo, baseadas na interligação das suas componentes, perante o cenário que se avizinha. Assumindo-se que a disponibilidade de mão de obra é também influenciada pelo seu preço, pretendemos, neste trabalho, identificar qual é o impacto das alterações do preço de mão de obra na sobrevivência das explorações. Tal previsão permitirá compreender melhor o comportamento coletivo e, daí, inferir sobre as atitudes a serem adotadas ou evitadas. 
Para simular o comportamento das explorações vitícolas, foram considerados vários intervenientes que influenciam o seu desempenho, recorrendo-se a modelos baseados em agentes (ABM). Os ABM constituem uma ferramenta que permite modelar com detalhes a forma como os indivíduos (agentes) respondem às circunstâncias externas (e. g., DeAngelis \& Mooij, 2005; Grimm et al., 2005; Railsback \& Grimm, 2012). Esses modelos são particularmente valiosos, de acordo com Galindro et al. (2018a), para sistemas não compreendidos o suficiente para construir um modelo baseado em equações (sistema de equações diferenciais, por exemplo). Os ABM mostram tal simplicidade que An et al. (2017) os referem como capazes de tratar representações abstratas da vida real que parecem muito complexas para a análise matemática tradicional. Outra característica interessante é que podem gerar resultados imprevisíveis, mas reveladores de padrões e relações antes não conhecidos (Grimm et al., 2005).

Os ABM têm sido aplicados numa ampla gama de vertentes, constituindo-se em uma ferramenta útil desde a Ecologia e Biologia às Ciências Sociais e Tecnológicas (Galindro et al., 2018a). No campo da economia, destaca-se o seu uso para resolver problemas latentes na economia (Farmer \& Foley, 2009); para descrever modelos de distribuição de renda enquadrados nas políticas de crédito e fiscais (Dosi et al., 2010, 2013); para a definição de incentivos financeiros em políticas orientadas ao mercado (Sierzchula et al., 2014; Shafiei et al., 2012); para a análise de ameaças à estabilidade financeira (Bookstaber, 2012). Em relação à economia agrícola, o uso de ABM tem aumentando progressivamente, nos últimos anos, para modelar os sistemas agrícolas, avaliar os fatores que afetam o processo de tomada de decisão e medir os impactos de políticas (Happe et al., 2005; Happe et al., 2008; Freeman et al., 2009; Nolan et al., 2009; Bert et al.; 2011; Balke \& Gilbert, 2014; MurrayRust et al., 2014; Groeneveld et al., 2017; Marvuglia et al., 2017; Huber et al. 2018; Kremmydas et al., 2018; Reidsma et al., 2018).

A análise política à escala das explorações agrícolas tem sido realizada mediante a utilização dos ABM, conforme exposto no trabalho de Kremmydas et al. (2018), dedicado a uma revisão sistemática de documentos publicados entre 2000 e 2016, e na análise de 184 documentos desenvolvida por Reidsma et al. (2018). Porém, se, por um lado, o uso dos ABM permite obter conhecimentos em nível desagregado e em escala espacial, proporcionando importantes aditamentos aos modelos tradicionais desenvolvidos no âmbito das explorações agrícolas (Ciaian et al., 2013), por outro, os ABM são frequentemente utilizados nos casos em que os agentes econômicos enfrentam capacidade limitada de informação e/ou processamento de informação e recursos finitos (Nolan et al., 2009). Tal situação torna vantajosa a modelização empírica por meio dos $A B M$, a qual reside na flexibilidade de especificação e concepção, na sua principal lacuna que exige procedimentos de verificação e validação (Parker et al., 2003).

Já Bousquet \& Le Page (2004) identificaram como as maiores vantagens dos ABM a combinação da sua natureza espacial e a capacidade de representar redes e, mais recentemente, Kremmydas et al. (2018) acrescentaram a sua capacidade de conjugar modelos sociais e ambientais, de estudar a emergência de respostas coletivas às políticas de gestão ambiental e a capacidade de modelar entidades individuais de tomada de decisão incorporando interações entre elas. Kremmydas et al. (2018) referem como principais desafios dos ABM novas perspectivas que os seus modelos permitem sobre sistemas complexos de recursos naturais e sobre a sua gestão, sendo necessário que a modelação das diversas interações seja integrada de forma direta e estabelecida com base em dados empíricos, utilizando-se, para isso, métodos estatisticamente sólidos.

O trabalho de Manson et al. (2016) é um exemplo de um modelo de rede social com ABM utilizado para adotar uma mudança sistêmica no sistema leiteiro dos Estados Unidos, construído com base em entrevistas realizadas com 53 agricultores. Neste trabalho, as entrevistas apoiam a definição e a estimativa de parâmetros para formalizar o processo de formação de vínculos com pares, instituições, organizações e grupos de pessoas. Outros exemplos de complementaridade de metodologias com ABM são disponibilizados nos trabalhos de Marvuglia et al. (2017) e Lan \& Yao (2019), tendo, nesses casos, sido utilizada a metodologia do life cycle assessment (LCA). No primeiro caso, foram utilizadas as respostas de 
168 agricultores e, no segundo, a amostra incluiu 1.000 explorações de um horizonte temporal de 30 anos.

Após uma breve introdução a este trabalho e a apresentação dos objetivos principais, 0 artigo encontra-se estruturado em mais três secção principais. A secção 2 apresenta a metodologia utilizada, com detalhes particulares no tipo de dados utilizados e na formulação de ABM obtida. A secção 3 apresenta simulação numérica e seus resultados. As conclusões gerais são finalmente apresentadas na secção 4.

\section{METODOLOGIA}

Para simular o comportamento das explorações vitícolas, recorreu-se aos ABM, considerando várias variáveis que influenciam a eficiência do setor. Essa metodologia foi adotada atendendo à complexidade do sistema agrícola, como as suas múltiplas escalas de interações (incluindo o ecossistema natural) e a sua forte influência no processo de tomada de decisão (Bert et al., 2014; Galindro et al., 2018a). Tais condições tornam as abordagens econométricas menos aplicáveis (Heckelei \& Britz, 2005), enquanto os ABM modelam interações reais entre as entidades (por exemplo, explorações agrícolas) e suas representações virtuais (Rounsevell et al., 2012; Galindro et al., 2018a). Os ABM consistem numa ferramenta adequada para melhorar a compreensão do comportamento dos agricultores em resposta às mudanças nas condições ambientais, econômicas ou institucionais, particularmente em âmbito local (An, 2012; Magliocca et al., 2015).

Neste trabalho, foram consideradas as vertentes ambiental (custos com fertilizantes, produtos fitofarmacêuticos, combustíveis, água e eletricidade), econômica (custos com amortizações) e também a social (custos reais com o trabalho) como centrais ao modelo definido, tendo em conta as condições institucionais que podem descrever a sustentabilidade a longo prazo da RDD em Portugal.

Como queremos avaliar a sustentabilidade das explorações vitícolas, considerando a heterogeneidade característica da RDD, cada exploração deve apresentar um conjunto de variáveis que exiba o seu atual status empreendedor, conforme procedimento desenvolvido em Galindro et al. (2018a). A premissa do modelo baseia-se num conjunto de características internas para cada exploração, como sua área, qualidade da uva, saldo bancário e produtividade. No processo de geração das explorações agrícolas, os valores considerados para essas caraterísticas vão ao encontro das informações recolhidas via inquérito presencial a 110 explorações vitícolas da RDD (Quadro 1).

Quadro 1. Breve descrição das 110 explorações inquiridas da RDD (valor médio e desvios padrão mínimo e máximo) relativa a 2017

\begin{tabular}{ccccc} 
Variável & Média & Desvio padrão & Mínimo & Máximo \\
Área de vinha (ha) & 17 & 30 & 1 & 184 \\
Produção de uva (kg/ha) & 5784 & 2661 & 1800 & 16531 \\
Produção de uva (€/ha) & 4315 & 1899 & 1312 & 11929 \\
Preço da uva (€/kg) & 0,77 & 0,4 & 0,2 & 1,6 \\
Subsídios recebidos (€/ha) & 440 & 296 & 59 & 1684 \\
Custos reais com o trabalho (€/ha) & 1868 & 1405 & 0 & 6798 \\
Custos com amortizações (€/ha) & 642 & 296 & 59 & 1684 \\
Custos com fertilizantes, & & & & 38 \\
fitofarmacêuticos, combustíveis, & 540 & 398 & & 3182 \\
eletricidade e água (€/ha) & & & 0,95 & 6,57 \\
Custos totais (€)/produto (kg) & 1,17 & & & \\
\hline
\end{tabular}

Fonte: dados próprios.

Em geral, a produtividade das explorações melhora com áreas maiores em razão dos crescentes retornos de escala (Sellers \& Alampi-Sottini, 2016). Para caracterizar a 
produtividade em função da área das explorações (Equação 1), foram usados os valores de produtividade por classe de área apresentados em Galindro et al. (2018b) e ajustados pelos seus valores médios.

$V(A)=\left\{\begin{array}{cc}-0,0007 A^{2}+0,0224 A+1,1813 & \text { se } A<25 \\ -0,0001 A+1,315 & \text { se } A \geq 25\end{array}\right.$

onde A representa a área da exploração, em ha.

No Quadro 2, apresentam-se as características das explorações geradas no que diz respeito à área, à qualidade da uva, ao saldo bancário e à produtividade. Os valores dessas variáveis aproximam-se dos observados na amostra (Quadro 1). De acordo com os valores observados na amostra, as áreas das explorações apresentam grandes desvios relativamente à média. Deste modo, optou-se por considerar dois grupos de área, G1 e G2, em que o grupo G2 é mais reduzido, correspondendo apenas a $8 \%$ das explorações e cujos valores de área são muito maiores do que os do grupo G1.

Quadro 2. Variáveis caracterizadoras das explorações - Condições iniciais

\begin{tabular}{|c|c|c|}
\hline Variável & Breve descrição & Condições iniciais \\
\hline $\begin{array}{c}\text { Preço de venda de } \\
\text { uvas }(P)\end{array}$ & Preço de venda da uva (€/kg) & $0,77 / 1,17$ \\
\hline Área $(A)$ & Área de vinha por exploração (ha) & $\begin{array}{c}G 1 \sim N(10.6,6.9), \\
\left.G 2 \sim N(30.6,20){ }^{*}\right)\end{array}$ \\
\hline Qualidade (Q) & $\begin{array}{l}\text { Condições internas da exploração (manejo, } \\
\text { terreno, estrutura da vinha) que podem } \\
\text { supervalorizar ou desvalorizar a produção }\end{array}$ & Random number (0.2-1.2) \\
\hline Saldo bancário (B) & Saldo bancário inicial (€/ha) & $B \sim N(43088,14458)$ \\
\hline Produtividade (V) & $\begin{array}{l}\text { Produtividade da exploração de acordo com o } \\
\text { seu tamanho }\end{array}$ & Equação 1 \\
\hline
\end{tabular}

Fonte: dados próprios. * G2 corresponde a 8\% das explorações.

Em cada período, a exploração produz e vende as suas uvas (para produção de vinho), considerando a própria capacidade produtiva e os preços no mercado externo: valor de venda da uva, mão de obra, consumo intermediário, entre outros. Posteriormente, o saldo bancário é atualizado, para avaliar se a exploração é sustentável e pode prosseguir com sua atividade, ou se o fecho é inevitável.

Para atingir um ambiente de simulação e aplicar a metodologia ABM, foram considerados os dados disponíveis na Rede de Informação de Contabilidades Agrícolas (20012017) relativos aos vinhos de qualidade do Douro. Por hectare, foram utilizados os valores de produção, em volume e valor; os subsídios auferidos pela atividade; os custos das amortizações como reveladores dos custos anuais de investimento; os custos do trabalho; uma variável ambiental que compila a soma dos custos com fertilizantes, produtos fitofarmacêuticos, combustíveis, eletricidade e água (todos especificados em euros). Considerando tais dados disponíveis de 2001 até 2017, foram ajustadas funções de Fourier para descrever o comportamento da região, tendo sido selecionadas aquelas que melhor se aproximam aos dados e que vão ao encontro das informações recolhidas nos inquéritos relativos ao ano de colheita de 2017 (Quadro 1), utilizando-o como ano-base. Esse procedimento foi desenvolvido por meio do software MATLAB. Posteriormente, a plataforma NetLogo foi usada para desenvolver ABM para simular os próximos 100 anos.

As variáveis do modelo, Trabalho (L), Ambiente $(E)$, Amortizações (Ca), Subsídios $(S)$ e Produção ( $($ ), partem dos valores obtidos em 2017 (condições iniciais) e evoluem ao longo do horizonte temporal, de acordo com as funções de Fourier obtidas: 


$$
\begin{aligned}
& L(t)=909,083+166,475 \cdot \cos \left(w_{1} t\right)+49,825 \cdot \sin \left(w_{1} t\right)+82,765 \cdot \cos \left(2 w_{1} t\right)+83,827 \cdot \sin \left(2 w_{1} t\right) \\
& E(t)=429,133+50,494 \cdot \cos \left(w_{2} t\right)-91,250 \cdot \sin \left(w_{2} t\right)-40,519 \cdot \cos \left(2 w_{2} t\right)-13,532 \cdot \sin \left(2 w_{2} t\right) \\
& C a(t)=563,524+78,314 \cdot \cos \left(w_{3} t\right)+152,478 \cdot \sin \left(w_{3} t\right) \\
& S(t)=367,409-93,275 \cdot \cos \left(w_{4} t\right)-129,906 \cdot \sin \left(w_{4} t\right)-43,685 \cdot \cos \left(2 w_{4} t\right) \\
& +20,841 \cdot \sin \left(2 w_{4} t\right)-16,475 \cdot \cos \left(3 w_{4} t\right)-22,025 \cdot \sin \left(3 w_{4} t\right) \\
& Y(t)=3828,7903+726,842 \cdot \cos \left(w_{5} t\right)+67,984 \cdot \sin \left(w_{5} t\right)+553,825 \cdot \cos \left(2 w_{5} t\right)+43,331 \cdot \sin \left(2 w_{5} t\right) \\
& +222,951 \cdot \cos \left(3 w_{5} t\right)+7,599 \cdot \sin \left(3 w_{5} t\right)+217.058 \cdot \cos \left(4 w_{5} t\right)-42,527 \cdot \sin \left(4 w_{5} t\right) \\
& -11,061 \cdot \cos \left(5 w_{5} t\right)+151,061 \cdot \sin \left(5 w_{5} t\right)
\end{aligned}
$$

Em que:

$w_{1}=0,3067 ; w_{2}=0,1742 ; w_{3}=0,2524 ; w_{4}=0,3158 ; w_{5}=0,3129$ e $t$ representa o período de tempo da simulação, com $t \in\{17,18, \ldots, 117\}$.

No Quadro 3, descrevem-se as variáveis utilizadas e as respetivas equações.

Quadro 3. Funções que descrevem as variáveis utilizadas no modelo ao longo do tempo

\begin{tabular}{ccc} 
Variável & Breve descrição & $\begin{array}{c}\text { Condiçães } \\
\text { iniciais }\end{array}$ \\
Trabalho $(L)$ & Custos de trabalho com a produção de uva $(€ / \mathrm{ha})$ & Equação 2 \\
Ambiente $(E)$ & Consumos intermédios com a produção de uva $(€ / \mathrm{ha})$ & Equação 3 \\
Amortizações $(C a)$ & Custos com amortizações anuais $(€ /$ ha) & Equação 4 \\
Subsídios $(S)$ & Ajudas monetárias à atividade atribuídas pelo & Equação 5 \\
Produção $(Y)$ & governo e União Europeia $(€ / \mathrm{ha})$ & Equação 6 \\
\hline
\end{tabular}

Fonte: dados próprios.

Neste contexto, foram considerados os seguintes pressupostos para identificar possíveis cenários futuros:

- Manutenção das tendências verificadas no período compreendido entre 2001 e 2017, por meio dos dados da Rede de Informação de Contabilidades Agrícolas (2001-2017), e sua aproximação aos dados recolhidos por inquérito, datados de 2017. Nessa situação, procedeu-se, ainda, à uniformização dos preços de acordo com os dados do Instituto Nacional de Estatística (2001-2017), considerando 2017 o ano-base.

- Introdução de um acréscimo no preço da mão de obra, para compensar a diminuição verificada na disponibilidade desse fator decorrente das dinâmicas demográficas, como envelhecimento da população (Fuchs et al., 2018), mas também a migração para áreas urbanas (van der Laan, 2016). Esse acréscimo foi calculado de acordo com as diminuições previstas na disponibilidade de mão de obra em função da evolução da população ativa, obtida por extrapolação das tendências nos últimos anos (Instituto Nacional de Estatística, 2017a, 2017b).

- Introdução de um acréscimo no preço de venda da uva capaz de remunerar os custos totais obtidos por unidade de produto. O cálculo desse novo preço baseou-se nas informações recolhidas nos inquéritos aos viticultores, localizado nos $1,17 € / \mathrm{kg}$, se considerados os custos totais da exploração, em que se incluem os custos atribuídos ao capital próprio e à mão de obra familiar. 


\subsection{Visão global do modelo}

\section{Entidades e variáveis de estado}

As 300 explorações vitícolas geradas possuem uma certa área (em ha), um valor de qualidade das uvas, um saldo bancário inicial e um valor de produtividade que depende da área. Todos esses valores foram gerados de acordo com a informação apresentada no Quadro 2, tentando ir ao encontro dos valores da amostra recolhida por meio de inquéritos presenciais ao viticultor (Quadro 1), para 2017.

\section{Produção}

A produção agrícola é o procedimento central desse modelo. Em cada período, as explorações realizam a sua atividade e ganham ou perdem dinheiro de acordo com a função Resultado $R_{i}(t)$, dada pela equação,

$$
R_{i}(t)=\left(Y(t) \cdot P(t) \cdot Q_{i} \cdot V_{i}\right) \cdot A_{i}+S(t) \cdot A_{i}-(E(t)+L(t)+C a(t)) \cdot A_{i}
$$

onde $i$ representa uma das 300 explorações, do conjunto $F=\{1, \ldots, 300\}$, com qualidade $Q_{i}$, produtividade $V_{i}$, área $A_{i} ; \mathrm{P}(\mathrm{t})$ representa o preço da uva no período $t$ e $L(t), E(t), C a(t), S(t)$ e $Y(t)$ correspondem aos valores das variáveis do modelo (Quadro 3 ) no período $t, t \in\{17, \cdots, 117\}$.

O resultado final atualiza o saldo bancário, $B_{i}(t)$, de cada uma das explorações em cada período de tempo, dado pela Equação 8, o que permite avaliar se a exploração é sustentável e capaz de realizar a sua atividade (procedimento de falência, de acordo com Galindro et al., 2018a).

$$
B_{i}(t)=B_{i}+\sum_{l=1}^{t} R_{i}(l)
$$

em que $B_{i}$ é o saldo bancário inicial da exploração $i, i \in F$.

\section{Falência}

Para avaliar a sustentabilidade das explorações vitícolas da RDD, testa-se o processo de falência, por meio do qual as explorações com saldo bancário abaixo de zero euro desaparecem do modelo.

\section{RESULTADOS}

O modelo inicial tenta obter informações sobre a evolução de 300 explorações. Cada uma das explorações é caracterizada pela área (A), qualidade $(\mathrm{Q})$, produtividade $(\mathrm{V})$ e saldo bancário (B), valores esses gerados aleatoriamente e de modo a reproduzir a realidade.

Para o preço da uva, consideram-se dois valores iniciais: 0,77 €/kg, que corresponde ao preço médio observado na amostra, e 1,17 €/kg, que representa os custos totais obtidos na amostra. Relativamente à mão de obra, distinguiram-se quatro cenários que correspondem, respetivamente, a um agravamento de $0 \%$ (manutenção da situação atual); agravamento de 0,2\% (variação da remuneração dos assalariados na agricultura, entre 2016 e 2017, Instituto Nacional de Estatística, 2017a); agravamento de 1\% (valor intermédio) e agravamento de 3\% (variação média da mão de obra utilizada entre 1989 e 2016, Instituto Nacional de Estatística, 2017a). A interface gráfica do nosso modelo (antes e depois de executar a simulação no NETLOGO 6.0.3) é apresentada nas Figuras 1 e 2, respetivamente, estando cada exploração representada por um círculo com tamanho proporcional à sua dimensão. A Figura 2 mostra a interface gráfica no final da simulação $(t=117)$, onde, nas condições estabelecidas, $24 \%$ das explorações da RDD estão fora do negócio. 


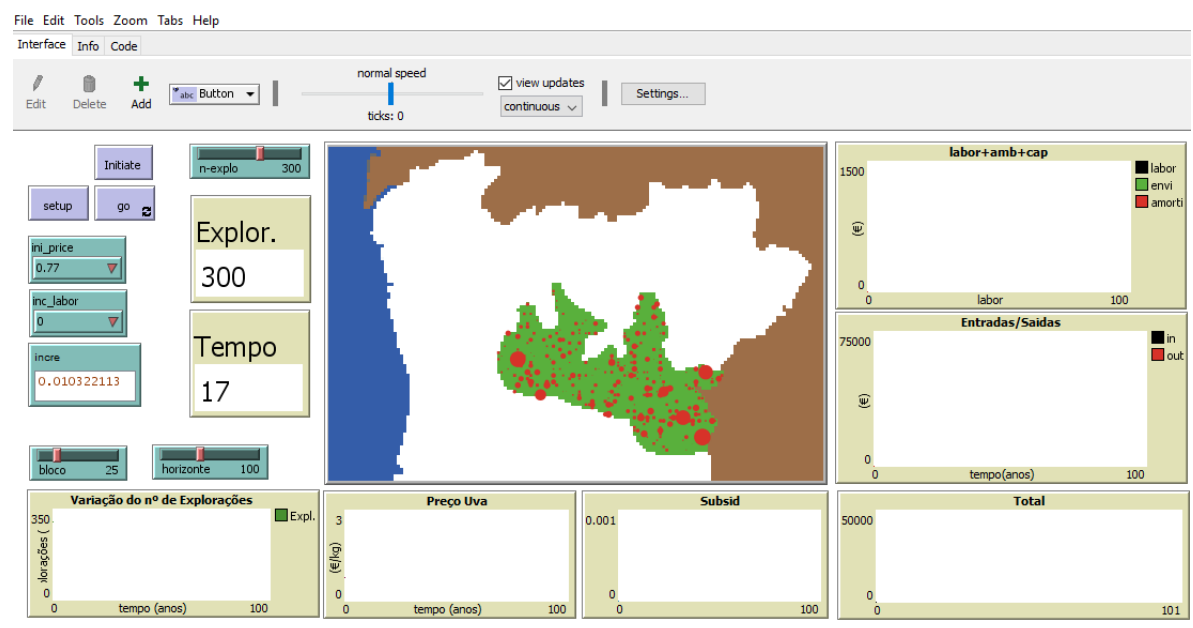

Figura 1. Interface gráfica do modelo de ABM na fase inicial $(t=17)$.

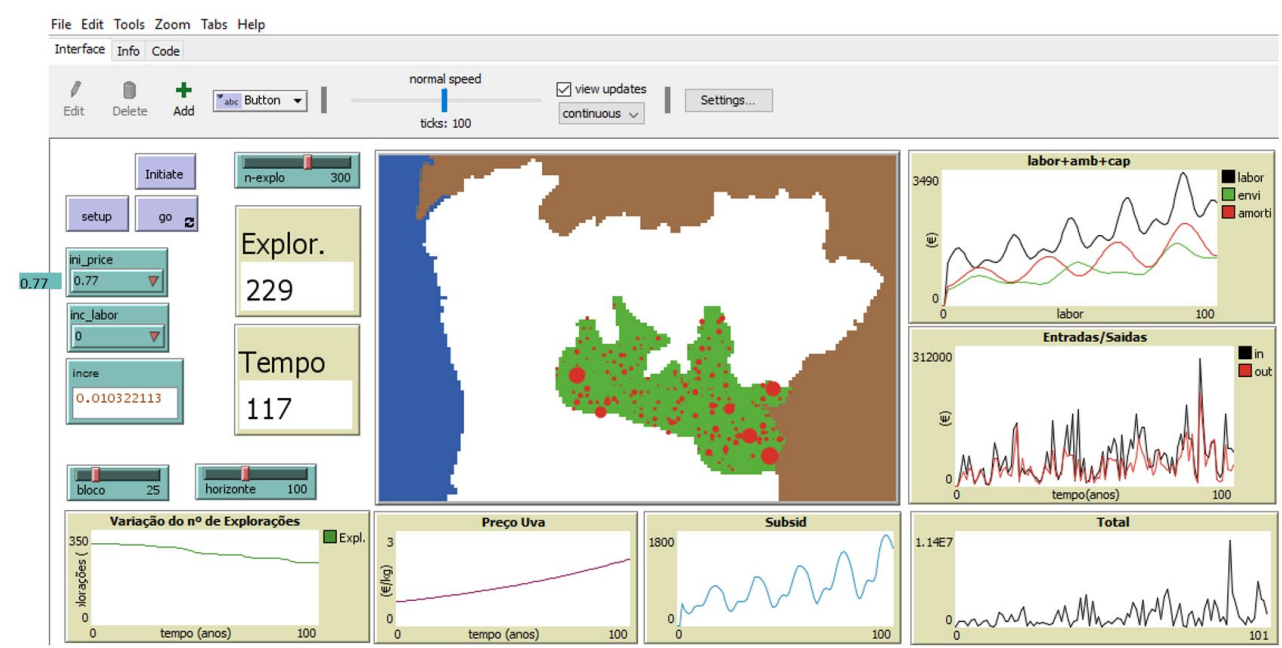

Figura 2. Interface gráfica do modelo de ABM na fase final $(t=117)$.

Por meio do modelo desenvolvido, foram testados os cenários apresentados anteriormente, de acordo com as perspectivas atuais cujos resultados são apresentados nos Quadros 4 e 5 e nas Figuras 3 e 4, respetivamente. Para cada cenário, o modelo é simulado dez vezes e os valores apresentados são valores médios. Considerou-se uma taxa de atualização de preços de 1,0103\% correspondente à média das taxas de variação do índice de preços no consumidor dos últimos 17 anos (Instituto Nacional de Estatística, 2001-2017). São apresentados os resultados, em termos de taxa de sobrevivência das explorações, ao fim de 25 anos, 50 anos, 75 anos e 100 anos.

Quadro 4. Taxa de sobrevivência a um preço inicial da uva de 0,77€/kg

\begin{tabular}{ccccc}
$\begin{array}{c}\text { Agravamento da } \\
\text { mão de obra }\end{array}$ & $\mathbf{3}$ & \multicolumn{3}{c}{ Anos decorridos } \\
\hline $0 \%$ & $98,42 \%$ & $\mathbf{5 0}$ & $\mathbf{7 5}$ & $\mathbf{1 0 0}$ \\
$0,2 \%$ & $98,57 \%$ & $80,93 \%$ & $77,05 \%$ \\
$1 \%$ & $95,45 \%$ & $85,82 \%$ & $77,55 \%$ & $72,30 \%$ \\
$3 \%$ & $71,30 \%$ & $71,70 \%$ & $56,55 \%$ & $42,28 \%$ \\
\hline
\end{tabular}

Fonte: dados próprios. 


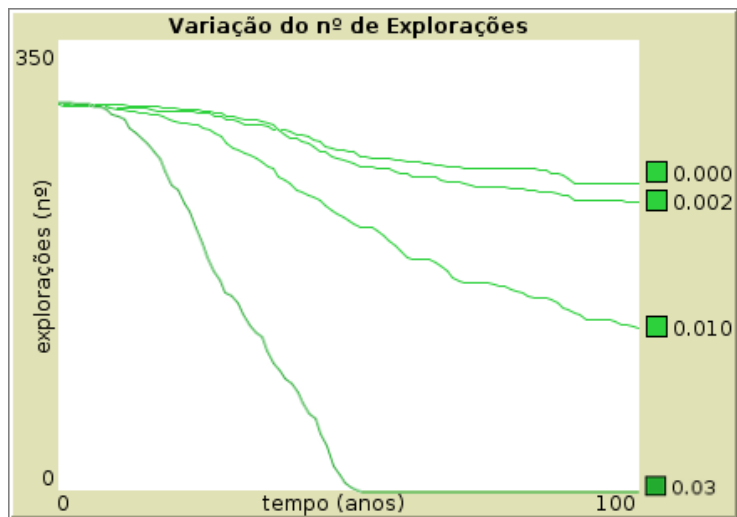

Figura 3. Número de explorações, na situação de preço inicial da uva de 0,77€/kg

Fonte: elaboração própria.

Considerando o preço inicial da uva, 0,77 €/kg (Quadro 4 e Figura 3), das 300 explorações existentes inicialmente, decorridos 25 anos, sobrevivem entre 214 e 295 explorações (entre $71,3 \%$ e $98,42 \%$ ), dependendo do agravamento no preço da mão de obra. Se alargarmos a um horizonte temporal de 100 anos, sobrevivem entre 127 e 231 explorações (entre 42,28\% e $77,05 \%)$, nos casos menos pessimistas de agravamento de mão de obra ( $0 \%, 0,2 \%$ e $1 \%)$.

Numa situação em que o agravamento no custo da mão de obra atinge os $3 \%$, decorridos 25 anos, desaparecem $28,7 \%$ das explorações, ao fim de 50 anos, $96,7 \%$ e, decorridos 75 anos, não existem explorações.

Quadro 5. Taxa de sobrevivência ao preço inicial da uva de 1,17€/kg

\begin{tabular}{ccccc}
$\begin{array}{c}\text { Agravamento da } \\
\text { mão de obra }\end{array}$ & $\mathbf{5}$ & $\mathbf{5 0}$ & $\mathbf{7 5}$ & $\mathbf{1 0 0}$ \\
\hline $0 \%$ & $99,72 \%$ & $98,37 \%$ & $95,70 \%$ & $93,18 \%$ \\
$0,2 \%$ & $99,65 \%$ & $97,33 \%$ & $93,12 \%$ & $89,95 \%$ \\
$1 \%$ & $99,12 \%$ & $89,93 \%$ & $80,47 \%$ & $72,15 \%$ \\
$3 \%$ & $89,40 \%$ & $46,47 \%$ & $0,03 \%$ & $0,00 \%$ \\
\hline
\end{tabular}

Fonte: dados próprios.

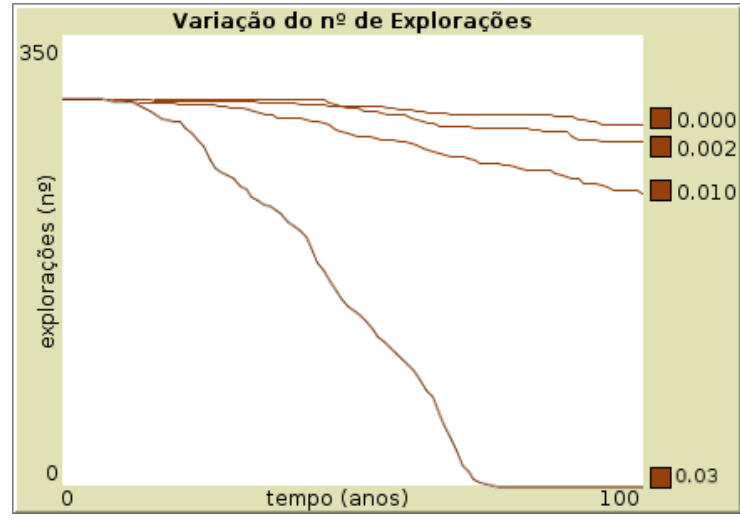

Figura 4. Número de explorações na situação de preço inicial da uva de 1,17€/kg.

Fonte: elaboração própria. 
Por seu lado, no caso de o preço da uva ser 1,17 €/kg (Quadro 5 e Figura 4), sobrevivem entre 268 e 299 explorações (entre 89,4\% e 99,72\%), em um prazo de 25 anos. Dependendo da variação do preço da mão de obra, com agravamento até $1 \%$, ao fim de 100 anos sobrevivem entre 216 e 280 explorações (entre $72,15 \%$ e 93,18\%).

Apesar de o agravamento no custo de mão de obra ser mais tolerado com esse novo preço de uva, continua a ser alarmante o cenário obtido decorridos 100 anos com agravamento de mão de obra de $3 \%$, no qual a situação de ausência de explorações parece inevitável. É de assinalar que tal perspectiva é inferior à redução de $5 \%$ verificada na força de trabalho, entre 2011 e 2016 (Instituto Nacional de Estatística, 2017b), mas muito próxima aos valores expectantes de - 2,8\% para a evolução da população ativa já para 2021 (Santos et al., 2019).

\section{CONCLUSÕES}

Neste artigo, foi apresentada a RDD, produtora de vinho português de qualidade. Após uma breve apresentação da relevância do setor vitivinícola para a economia portuguesa, a influência do preço da mão de obra na análise da sustentabilidade do setor na RDD foi selecionada como o principal objetivo deste artigo. A tendência da atividade foi simulada por meio dos ABM, avaliando-se a sobrevivência das explorações na região para os cenários alternativos selecionados. O modelo teve como pressupostos essenciais a geração aleatória e o ajuste de funções dependentes do tempo baseadas em dados reais.

No final do horizonte de simulação (após 100 anos), das 300 explorações existentes inicialmente, sobrevivem entre 127 e 280 (entre $42,28 \%$ e 93,18\%), dependendo do preço de venda da uva e atendendo a flutuações no custo da mão de obra de até $1 \%$.

Considerando o nosso modelo de formulação e as premissas definidas, verifica-se que um simples acréscimo do preço de venda de uva de $0,4 €(1,17 €-0,77 €)$ na RDD pode evitar a extinção de cerca de $30 \%$ das explorações, num horizonte de 100 anos, porém continua a verificar-se o desaparecimento de $27,85 \%$, considerando uma flutuação no preço de mão de obra de até $1 \%$.

Cenários de variações de custo da mão de obra acima desse valor põem em causa a sobrevivência das explorações num horizonte temporal mais amplo.

De modo geral, os resultados mostram que a RDD não está bem preparada para lidar com os cenários negativos que se estimam poder verificar-se, conduzindo a um esgotamento grave das taxas de sobrevivência das explorações agrícolas durante as experiências desenvolvidas. O preço a que a uva vem sendo valorizada no mercado e o custo e disponibilidade de mão de obra devem ser objeto rápido de intervenção no setor, para garantir a sua sustentabilidade na região. Note que se trata de uma região cujo relevo impõe o recurso intenso de mão de obra para a manutenção da atividade.

\section{Agradecimentos}

Este trabalho é suportado pelo Projeto de I\&D - INNOVINE \& WINE - Plataforma de Inovação da Vinha e do Vinho - Operação NORTE -01-0145-FEDER-000038, co-financiado pelos Fundos Europeus Estruturais e de Investimento (FEDER) e pelo NORTE2020 (Programa Operacional Regional do Norte 2014/2020).

\section{REFERÊNCIAS BIBLIOGRÁFICAS}

An, G., Fitzpatrick, B. G., Christley, S., Federico, P., Kanarek, A., Miller-Neilan, R., Oremland, M., Salinas, R., Laubenbacher, R., \& Lenhart, S. (2017). Optimization and control of agent-based models in biology: a perspective. Bulletin of Mathematical Biology, 79(1), 63-87.

An, L. (2012). Modeling human decision in coupled human and natural systems: review of agent-based models. Ecological Modelling, 229, 25-36.

Balke, T., \& Gilbert, N. (2014). How do agents make decisions? A survey. Journal of Artificial Societies and Social Simulation, $17(13)$ 
Bert, F. E., Podestá, G. P., Rovere, S. L., Menéndez, Á. N., North, M., Tatara, E., Laciana, C. E., Weber, E., \& Toranzo, F. R. (2011). An agent based model to simulate structural and land use changes in agricultural systems of the Argentine Pampas. Ecological Modelling, 222, 3486-3499.

Bert, F. E., Rovere, S. L., Macal, C. M., North, M. J., \& Podestá, G. P. (2014). Lessons from a comprehensive validation of an agent based-model: The experience of the Pampas Model of Argentinean agricultural systems. Ecological Modelling, 273, 284-298.

Bookstaber, R.M. (2012). Using agent-based models for analyzing threats to financial stability. Office of Financial Research.

Bousquet, F., \& Le Page, C. (2004). Multi-agent simulations and ecosystem management: a review. Ecological Modelling, 176(3-4), 313-332.

Ciaian, P., Espinosa, M., Paloma, S. G., Heckelei, T., Langrell, S., Louhichi, K., Sckokai, P., Thomas, A., \& Vard, T. (2013). Farm level modelling of CAP: A methodological overview. In: European Commission. JRC Scientific and Policy Report. EC: Seville, Spain. Recuperado em 18 de outubro de 2019, de http://publications.jrc.ec.europa.eu/repository/handle/JRC79969

Deangelis, D. L., \& Mooij, W. M. (2005). Individual-based modeling of ecological and evolutionary processes. Annual Review of Ecology Evolution and Systematics, 36, 147-168.

Dosi, G., Fagiolo, G., Napoletano, M., \& Roventini, A. (2013). Income distribution, credit and fiscal policies in an agent-based keynesian model. Journal of Economic Dynamics \& Control, 37, 1598-1625.

Dosi, G., Fagiolo, G., \& Roventini, A. (2010). Schumpeter meeting keynes: a policy-friendly model of endogenous growth and business cycles. Journal of Economic Dynamics \& Control, 34, 1598-1625.

Farmer, J. D., \& Foley, D. (2009). The Economy needs agent-based modelling. Nature, 460, 685-686.

Flint, D. J., Golicic, S. L., \& Signori, P. (2011, June 8-11). Sustainability through resilience: the very essence of the wine industry. In 6th AWBR International Conference, Bordeaux Management School, France.

Freeman, T., Nolan, J., \& Schoney, R. (2009). An agent-based simulation model of structural change in Canadian Prairie Agriculture, 1960-2000. Canadian Journal of Agricultural Economics, 57, 537-554.

Fuchs, J., Söhnlein, D., Weber, B., \& Weber, E. (2018). Stochastic Forecasting of Labor Supply and Population: An Integrated Model. Population Research and Policy Review, 37(1), 37-58.

Galindro, A., Marta-Costa, A., Santos, C., Matias, J., \& Cerveira, A. (2018a). Simulation of alternative scenarios in Portuguese wine sector: An Agent-based model approach. In D. Vrontis, Y. Weber \& E. Tsoukatos (Eds.), Proceedings of the 11th Annual Conference of the EuroMed Academy of Business (EMAB) "Research Advancements in National and Global Business Theory and Practice" (pp. 544-555), Valletta, Malta.

Galindro, A., Santos, M., Santos, C., Marta-Costa, A., Matias, J. \& Cerveira, A. (2018b). Wine productivity per farm size: A maximum entropy application. Wine Economics and Policy, 7(1), 77-84. 10.1016/j.wep.2018.03.001.

Grimm, V., Revilla, E., Berger, U., Jeltsch, F., Mooij, W. M., Railsback, S. F., Thulke, H. H., Weiner, J., Wiegand, T., \& Deangelis, D. L. (2005). Pattern-oriented modeling of agent-based complex systems: lessons from ecology. Science, 310, 987-991.

Groeneveld, J., Müller, B., Buchmann, C. M., Dressler, G., Guo, C., Hase, N., Hoffmann, F., John, F., Klassert, C., Lauf, T., Liebelt, V., Nolzen, H., Pannicke, N., Schulze, J., Weise, H., \& Schwarz, N. (2017). Theoretical foundations of human decision-making in agent - based land use models - A Review. Environmental Modelling \& Software, 87, 39-48.

Hall, C. M., \& Sharples, L. (2008). Food and wine festivals and events around the world: development, management and markets. Oxford, Elsevier/Butterworth-Heinemann

Happe, K., Balmann, A., Kellermann, K., \& Sahrbacher, C. (2005). The use of agent-based modelling to establish a link between agricultural policy reform and structural change. In F. Arfini (Ed.), Modelling agricultural policies: state of the art and new challenges (pp. 138-165). Parma, Monte Universit `a Parma Editore.

Happe, K., Balmann, A., Kellermann, K., \& Sahrbacher, C. (2008). Does Structure Matter? The Impact of Switching the Agricultural Policy Regime on Farm Structures. Journal of Economic Behavior \& Organization, 67, 431-444.

Heckelei, T., \& Britz, W. (2005). Models based on positive mathematical programming: State of the art and further extensions. In F. Arfini (Ed.). Modelling agricultural policies: State of the art and new challenges (pp. 48-73). Parma, Monte Universita Parma Editore.

Huber, R., Bakker, M., Balmann, A., Berger, T., Bithell, M., Brown, C., Grêt-Regamey, A., Xiong, H., Le, Q. B., Mack, G., Meyfroidt, P., Millington, J., Müller, B., Polhill, J. G., Sun, Z., Seidl, R., Troost, C., \& Finger, 
R. (2018). Representation of Decision-Making in European Agricultural Agent-Based Models. Agricultural Systems, 167, 143-160.

Instituto Nacional de Estatística - INE (2017a). Inquérito à Estrutura das Explorações Agrícolas. Recuperado em 23 de setembro de 2019, de http://www.ine.pt/

Instituto Nacional de Estatística - INE (2017b). População residente $\left(\right.$ ( $^{\circ}{ }^{\circ}$ por Local de residência (NUTS 2013), Sexo e Grupo etário; Estimativas anuais da população residente. Recuperado em 20 de outubro de 2019, de http://www.ine.pt/

Instituto Nacional de Estatística - INE (2001-2017). Taxa de Variação do Índice de Preços no Consumidor. Recuperado em 20 de maio de 2019, de http://www.ine.pt/

Instituto da Vinha e do Vinho - IVV (2018). Evolução da Área Total de Vinha - Portugal Continental. Recuperado em 05 de junho de 2019, de https://www.ivv.gov.pt/np4/35/ [[Q8: Q8]]

Kremmydas, D., Athanasiadis, I. N., \& Rozakis, S. (2018). A review of agent based modeling for agricultural policy evaluation. Agricultural Systems, 164, 95-106.

Lan, K., \& Yao, Y. (2019). Integrating life cycle assessment and agent-based modeling: a dynamic modeling framework for sustainable agricultural systems. Journal of Cleaner Production, 238, 238.

Magliocca, N., Mcconnell, V., \& Walls, M. (2015). Exploring Sprawl: Results from an agent-based model of land and housing markets. Ecological Economics, 113, 114-125.

Manson, S. M., Jordan, N. R., Nelson, K. C., \& Brummel, R. F. (2016). Modeling the effect of social networks on adoption of multifuncional Agriculture. Environmental Modelling \& Software, 75, 388401.

Marta-Costa, A., Torres-Manso, F., \& Tibério, L. (2012). Sustainability diagnosis of an agroforestry system. Regional Science Inquiry Journal, IV(2), 111-124.

Marta-Costa, A. A. (2010). Application of decision support methods for sustainable agrarian systems. New Medit, 9(2), 42-49.

Marvuglia, A., Rege, S., Navarrete Gutiérrez, T., Vanni, L., Stilmant, D., \& Benetto, E. (2017). A return on experience from the application of agent-based simulations coupled with life cycle assessment to model agricultural processes. Journal of Cleaner Production, 142, 1539-1551.

Mencarelli, F., \& De Propris, L. (2014). Mappa dei modelli di produzione sostenibile: gli indicatori della sostenibilità del vino. In: Forum per la Sostenibilitàdel Vino (Ed.), Primo Rapporto sulla Sostenibilitò del Vino (pp. 35-61). Unione Italiana Vini.

Moreira, N., \& Guedes de Pinho, P. (2011). Port wine. In R.S Jackson (Ed.), Advances in food and nutrition research. (Vol. 63). USA: Elsevier Inc.

Murray-Rust, D., Robinson, D. T., Guillem, E., Karali, E., \& Rounsevell, M. (2014). An open framework for agent based modelling of agricultural land use change. Environmental Modelling \& Software, 61, 1938.

Nolan, J., Parker, D., Van Kooten, G. C., \& Berger, T. (2009). An overview of computational modeling in agricultural and resource economics. Canadian Journal of Agricultural Economics, 57, 417-429.

Parker, D. C., Manson, S. M., Janssen, M. A., Hoffmann, M. J., \& Deadman, P. (2003). Multiagent systems for the simulation of land-use and land-cover change: a review. Annals of the Association of American Geographers, 93(2), 314-337.

International Organization of Vine \& Wine - OIV. (2019). OIV Statistical Report on World Vitiviniculture: World Vitiviniculture Situation. Recuperado em 01 de junho de 2019, de http://www.oiv.int/public/medias/6782/oiv-2019-statistical-report-on-world-vitiviniculture.pdf

Rachão, S., \& Joukes, V. (2017). Co-creating the branding of rural destinations: an analysis of agritourism facebook pages in the northern demarcated wine regions of Portugal. Revista Turismo \& Desenvolvimento, 1(27-28), 1933-1946.

Railsback, S. F., \& Grimm, V. (2012). Agent-Based and Individual-Based Modeling: A Practical Introduction. New Jersey: Princeton University Press.

Rebelo, J. (2018). Rentabilidade das explorações vitícolas: a relevância do fator trabalho. In T. Hogg \& J Rebelo (Eds.), Rumo Estratégico para o setor dos vinhos do Douro e Porto, Volume 3 - Estudos de base (pp. 119-121). Vila Real: UTAD.

Reidsma, P., Janssen, S., Jansen, J. \& van Ittersum, M.K. (2018). On the development and use of farm models for policy impact assessment in the European Union - A review. Agricultural Systems, 159, 111-125. 
Rede de Informação de Contabilidades Agrícolas - RICA. (2001-2017), Resultados médios por exploração, Recuperado em 13 de março de 2019, de http://www.gpp.pt/index.php/rica/rede-de-informacaode-contabilidades-agricolas-rica

Rounsevell, M. D. A., Robinson, D. T., \& Murray-Rust, D. (2012). From actors to agents in socio-ecological systems models. Biological Sciences, 367, 259-269.

Santos, C., Galindro, A., \& Marta-Costa, A. (2019). The Labour Market Impact in the Douro Viticulture: A Fuzzy cluster distribution approach. Regional Science Inquiry Journal, 11(2), 143-151.

Sellers, R., \& Alampi-Sottini, V. (2016). The influence of size on winery performance: Evidence from Italy. Wine Economics and Policy, 5(1), 33-41.

Shafiei, E., Thorkelsson, H., Ásgeirsson, E., Davidsdottir, B., Raberto, M., \& Stefansson, H. (2012). An agent-based modeling approach to predict the evolution of market share of electric vehicles: A case study from Iceland. Technological Forecasting and Social Change, 79, 1638-1653.

Sierzchula, W., Bakker, S., Maat, K., \& Van Wee, B. (2014). The Influence of Financial Incentives and Other Socio-Economic Factors on Electric Vehicle Adoption. Energy Policy, 68, 183-194.

Van Der Laan, R. J. (2016). Identifying key factors influencing sustainable wine production - A case study on the wine sector in the Porto and Douro region, Portugal (Master thesis). Utrecht University, Utrecht The Netherlands.

Viassone, M., Vrontis, D., \& Papasolomou, I. (2016). The Relationship Between Wine Sector and Regional Competitiveness. Global Business and Economics Review, 18(3-4), 259-276.

Submetido em: 18/10/2019

Aceito em: 19/05/2020

Classificação JEL: C63, Q12. 